\title{
The TanDEM-X Mission: A Satellite Formation for High-Resolution SAR Interferometry
}

\author{
G. Krieger, H. Fiedler, M. Zink, I. Hajnsek, M. Younis, S. Huber, M. Bachmann, J. Hueso Gonzalez, M. Werner, A. Moreira \\ Microwaves and Radar Institute, German Aerospace Center (DLR), 82234 Oberpfaffenhofen, Germany \\ Gerhard.Kriegeradr.de
}

\begin{abstract}
TanDEM-X (TerraSAR-X add-on for Digital Elevation Measurements) is an innovative spaceborne radar interferometer mission that was approved for full implementation by the German Space Agency in spring 2006. This paper gives an overview of the TanDEM-X mission concept, summarizes the basic products, illustrates the achievable performance, and provides some examples for new imaging modes and applications.
\end{abstract}

\section{INTRODUCTION}

The primary objective of the TanDEM-X mission is the generation of a world-wide, consistent, timely, and high precision digital elevation model aligned with the HRTI-3 specification as the basis for a wide range of scientific research, as well as for commercial DEM production [1]. This goal will be achieved by means of a second, TerraSAR-X like satellite flying in close orbit configuration with TerraSAR-X. Both satellites will then act as a large single-pass SAR interferometer with the opportunity for flexible baseline selection. This enables the acquisition of highly accurate cross-track and along-track interferograms without the inherent accuracy limitations imposed by repeat-pass interferometry due to temporal decorrelation and atmospheric disturbances. Besides the primary goal of the mission, several secondary mission objectives based on along-track interferometry as well as new techniques with bistatic SAR have been defined which represent an important and innovative asset of the mission. TanDEM-X will be implemented in the framework of a public-private partnership between the German Aerospace Center (DLR) and EADS Astrium $\mathrm{GmbH}$, as for TerraSAR-X. The launch of TanDEM-X is planned for spring 2009.

\section{Mission CONCEPT}

The TanDEM-X mission is an extension of the TerraSAR-X mission, co-flying a second satellite of nearly identical capability in a close formation. The TerraSAR-X satellite (TSX), as basis for TanDEM-X, is not only a high performance SAR system with respect to SAR image and operational features, but it has already built in all necessary features required for the implementation of the TanDEM-X mission. Examples are additional X-band horn antennas for inter-satellite phase synchronization, the availability of a dual-frequency GPS receiver for precise orbit determination, excellent $\mathrm{RF}$ phase stability of the SAR instrument, and PRF synchronization based on GPS as a common time reference. The second satellite (TDX) will be as much as possible a rebuild of TSX with only minor modifications like an additional cold gas propulsion system for formation fine tuning and an additional S-band receiver to enable the reception of status and GPS position information broadcasted by TSX. This guarantees a low development risk and it offers the possibility for a flexible share of operational functions among the two satellites. The TDX satellite will be designed for a nominal lifetime of $5 \frac{1}{2}$ years and has a nominal overlap with TSX of 3 years. Note in this context that TSX holds consumables and resources for up to seven years of operation, allowing for a potential prolongation of the overlap and the TanDEM-X mission duration.

The instruments on both satellites are advanced high resolution X-band synthetic aperture radars based on active phased array technology, which can be operated in Spotlight, Stripmap, and ScanSAR mode with full polarization capability [2]. The center frequency of the instruments is $9.65 \mathrm{GHz}$ with a selectable SAR chirp bandwidth of up to $300 \mathrm{MHz}$. The active phased array antenna, which has an overall aperture size of 4.8 $\mathrm{m} \times 0.7 \mathrm{~m}$, is fixed mounted to the spacecraft body and incorporates 12 panels with 32 dual-pol waveguide sub-arrays each. This enables agile beam pointing and flexible beam shaping.

\section{A. Interferometric Data Acquisition}

Interferometric data acquisition with the TanDEM-X satellite formation can be achieved in different operational modes. Examples are Bistatic, Monostatic, and Alternating Bistatic operation, which are illustrated in Fig. 1. The three interferometric configurations may further be combined with different TSX and TDX SAR imaging modes like Stripmap, ScanSAR, Spotlight, and Sliding Spotlight. Operational DEM generation is planned to be performed using the bistatic InSAR Stripmap mode shown in Fig. 1 in the middle. This mode uses either TSX or TDX as a transmitter to illuminate a common radar footprint on the Earth's surface. The scattered signal is then recorded by both satellites simultaneously. This simultaneous data acquisition makes dual use of the available transmit power and is mandatory to avoid possible errors from temporal decorrelation and atmospheric disturbances.

\section{B. Relative Phase Referencing}

A peculiarity of the bistatic data acquisition is the use of independent oscillators for modulation and demodulation of the radar pulses. Any deviation between the two oscillators will hence cause a residual modulation of the recorded azimuth signal. The impact of oscillator phase noise in bistatic SAR has been analyzed in [3] where it is shown that oscillator noise may cause significant errors in both the interferometric phase and SAR focusing. The stringent requirements for interferometric phase stability in the bistatic mode will hence require an appropriate relative phase referencing between the two SAR instruments or an operation in the alternating bistatic mode. For TanDEM-X, a dedicated inter-satellite X-band synchronization link will be established by a mutual exchange of radar pulses between the two satellites. For this, the nominal bistatic SAR data acquisition is shortly interrupted, and a radar pulse is redirected from the main SAR antenna to one of six dedicated synchronization horn antennas mounted on each spacecraft. The pulse is then recorded by the other satellite which in turn transmits a short synchronization pulse. By this, a bidirectional link between the two radar instruments will be established, which allows for mutual phase referencing without exact 

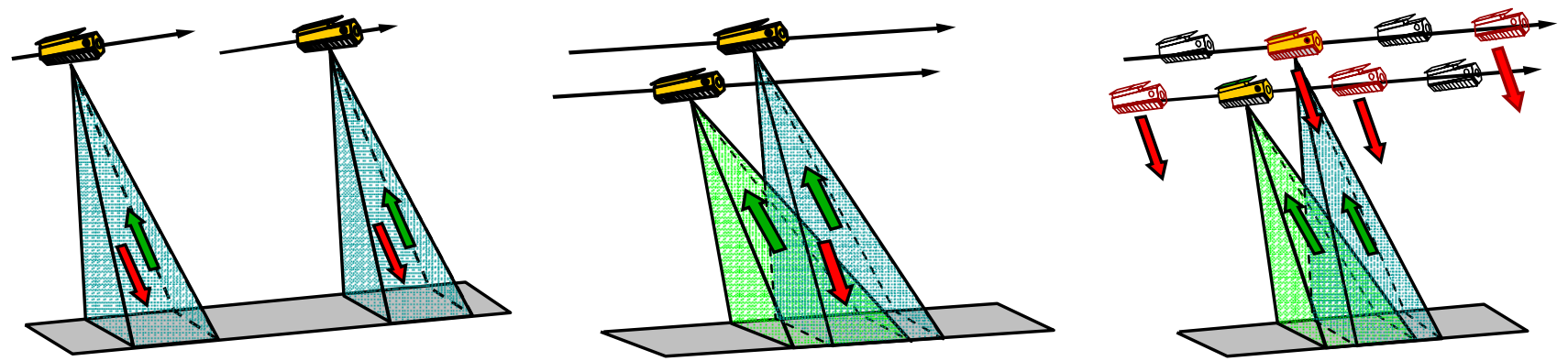

Fig. 1: Data acquisition modes for TanDEM-X: Pursuit monostatic (left), bistatic (middle), alternating bistatic (right).

knowledge of the actual distance between the satellites. On ground, a correction signal can then be derived from the recorded synchronization pulses which compensates the oscillator induced phase errors in the bistatic SAR signal. The performance of such a synchronization link has been investigated in [4]. Fig. 2 shows the predicted standard deviation of the residual phase errors after synchronization as a function of the update frequency of the synchronization signals for different signal-to-noise ratios (SNR) of the bidirectional link. The actual SNR varies with the distance between the satellites as well as their relative attitude. For the typical DEM data acquisition mode with baselines below $1 \mathrm{~km}$, the SNR will be in the order of 30 to $40 \mathrm{~dB}$, and it becomes clear that a phase error below $1^{\circ}$ can be achieved for synchronization frequencies of $5 \mathrm{~Hz}$.

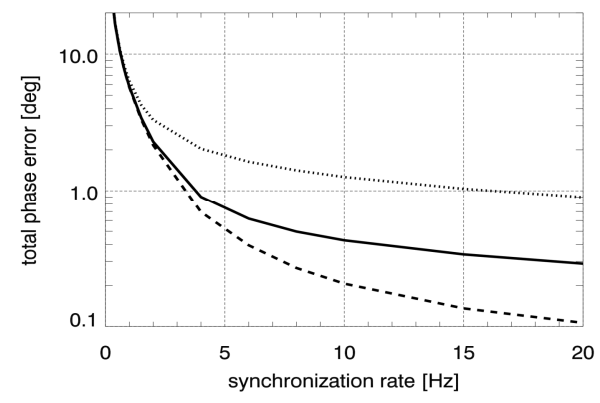

Fig. 2: Standard deviation of the total synchronization link phase error as a function of the synchronization frequency. The three curves are for signal-tonoise ratios of $20 \mathrm{~dB}$ (dotted), $30 \mathrm{~dB}$ (solid), and $40 \mathrm{~dB}$ (dashed). The standard deviation should be $1^{\circ}$ to meet the TanDEM-X requirements.

\section{Orbit Configuration and Formation Flying}

The TanDEM-X operational scenario requires the coordinated operation of two satellites flying in close formation. The adjustment parameters for the formation are the orbits node line angle, the angle between the perigees, the orbit eccentricities and the phasing between the satellites. With these parameters, several options have been investigated during the phase A study, and the HELIX satellite formation shown in Figure 3 has finally been selected for operational DEM generation. This formation combines an out-of-plane (horizontal) orbital displacement by different ascending nodes with a radial (vertical) separation by different eccentricity vectors resulting in a helix like relative movement of the satellites along the orbit. Since there exists no crossing of the satellite orbits, arbitrary shifts of the satellites along their orbits are allowed. This enables a safe spacecraft operation without the necessity for autonomous control. It is furthermore possible to optimize the along-track displacement at predefined latitudes for different applications: cross-track interferometry will aim at along-track baselines which are as short as possible to ensure an optimum overlap of the Doppler spectra and to avoid temporal decorrelation in vegetated areas, while other applications like along-track interferometry or super resolution require selectable along-track baselines in the range from hundred meters up to several kilometers. A fine tuning of the satellite formation will be performed via the aforementioned cold gas propulsion system on TDX.

The HELIX formation enables a complete mapping of the Earth with a stable height of ambiguity by using a small number of formation settings [5]. Southern and northern latitudes can be mapped with the same formation setting by using ascending orbits for one and descending orbits for the other hemisphere, as illustrated in Figure 3 on the right. A fine tuning of the crosstrack baselines can be achieved by taking advantage of the natural rotation of the eccentricity vectors due to secular disturbances, also called motion of libration. The phases of this libration can be kept in a fixed relative position with small manoeuvres using the cold gas thrusters on a daily basis, while major formation changes as well as a duplication of the orbit keeping manoeuvres required by TSX will be performed by the hot gas thrusters.
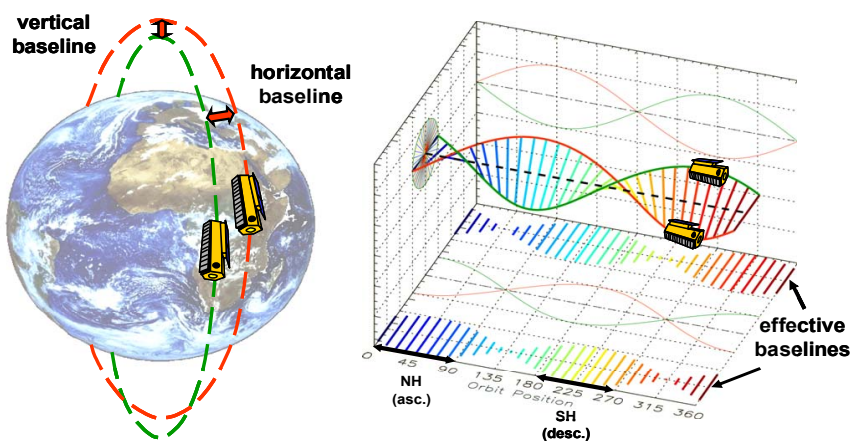

Fig. 3: HELIX satellite formation for TanDEM-X. Left: orbital arrangement. Right: cross-track baselines.

\section{PERFormanCE ANALYSIS}

This section investigates the interferometric performance of TanDEM-X. For this, an interferometric data acquisition in bistatic Stripmap mode will be assumed. Major factors which affect the relative height accuracy are the radiometric sensitivity of each SAR instrument, range and azimuth ambiguities, quantization noise, processing and co-registration errors as well as surface and volume decorrelation, scaled by the baseline length [5]. The key quantity in estimating the interferometric performance is the coherence which has been computed by the product

$$
\gamma_{\text {tot }}=\gamma_{S N R} \cdot \gamma_{\text {Quant }} \cdot \gamma_{A m b} \cdot \gamma_{\text {Coreg }} \cdot \gamma_{\text {Geo }} \cdot \gamma_{A z} \cdot \gamma_{V o l} \cdot \gamma_{\text {Temp }}
$$

where the right hand side describes the different error contributions due to the limited $\operatorname{SNR}\left(\gamma_{S N R}\right)$, quantization $\left(\gamma_{\text {Quant }}\right)$, ambiguities $\left(\gamma_{A m b}\right)$, limited coregistration accuracy $\left(\gamma_{\text {Coreg }}\right)$, baseline decorrelation $\left(\gamma_{\text {Geo }}\right)$, relative shift of Doppler spectra $\left(\gamma_{A z}\right)$, volume decorrelation $\left(\gamma_{V o l}\right)$, and temporal decorrelation $\left(\gamma_{\text {Temp }}\right)$. Each of these terms has been evaluated and Fig. 4 shows 
the result of the interferometric performance analysis for two different ambiguous heights corresponding to different baseline lengths in the order of $300 \mathrm{~m}$ and $450 \mathrm{~m}$, respectively.

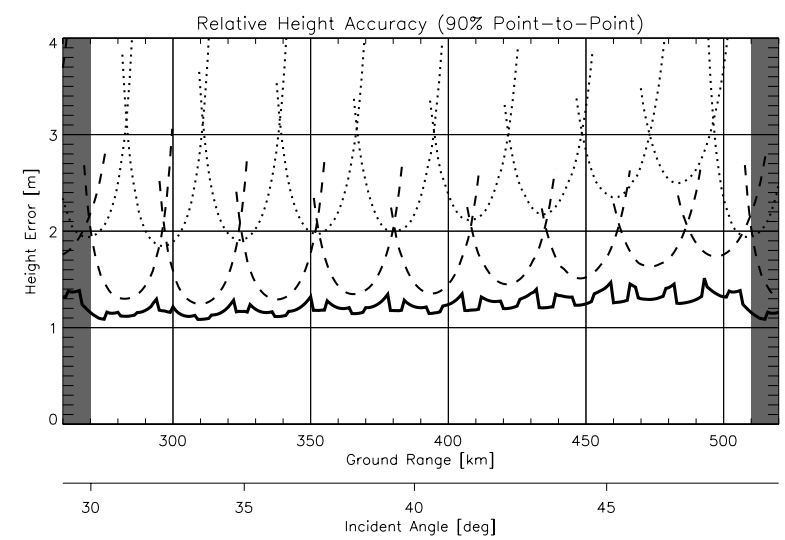

Fig. 4: Height accuracy for a height of ambiguity of $45 \mathrm{~m}$ (dotted) and $30 \mathrm{~m}$ (solid). The lower solid curve shows the error resulting from the combination of multiple swaths. All errors are point-to-point height errors for a $90 \%$ confidence interval.

\section{NEW IMAGING TECHNIQUES}

The TanDEM-X mission will provide the remote sensing scientific community with a unique data set to exploit the capability of new bistatic radar techniques and to apply these innovative techniques for enhanced geo- and biophysical parameter retrieval.

\section{A. Along-Track Interferometry}

TanDEM-X is predestined for along-track interferometry (ATI), which compares the phase of two complex SAR images acquired in identical geometries but separated by a short time interval. This technique is well suited for monitoring dynamic processes on the Earth's surface. As outlined in Section 2, it is possible to adjust the along-track displacement between the two satellites from almost zero to several kilometers. By this, it becomes possible to adapt the ATI sensitivity of TanDEM-X to a wide range of radial velocities. The HELIX formation enables even a minimization of the across-track component for a given latitude and incident angle. Along-track interferometry can furthermore be performed by the so-called dual-receive antenna mode in each of the two tandem satellites, which provides additional along-track baselines of $2.4 \mathrm{~m}$ [10]. The combination of short and long baseline ATI data acquisitions can be used to improve both the detection and localisation of moving objects and to resolve phase ambiguities from high-velocity scatterers. TanDEM-X provides hence a capable along-track SAR interferometer with four phase centers. Potential applications are Ground Moving Target Indication (GMTI), the measurement of ocean currents, and the monitoring of sea ice drift.

\section{B. Very Large Baseline Cross-Track Interferometry}

Very large baseline interferometry takes advantage of the high RF bandwidth of the TSX and TDX satellites, which allows for coherent data acquisitions with baselines of up to 5 $\mathrm{km}$ and more. Note that less than $5 \%$ of the maximum possible (critical) baseline length is used during nominal DEM data acquisition. Large baseline interferograms can hence significantly improve the height accuracy, but the associated low height of ambiguity requires a combination of multiple interferograms with different baseline lengths to resolve phase ambiguities. By this, it becomes possible to derive DEMs with
HRTI-4 like accuracy on a local or even regional scale. Further opportunities arise from a comparison of multiple large baseline TanDEM-X interferograms acquired during different passes of the satellite formation. This provides a very sensitive measure for vertical scene and structure changes. Potential applications are a detection of the grounding line which separates the shelf from the inland ice in polar regions, monitoring of vegetation growth, mapping of atmospheric water vapour with high spatial resolution, measurement of snow accumulation or the detection of anthropogenic changes of the environment, e.g. due to deforestation. Note that most of these combinations rely on a comparison of two or more single-pass (large baseline) crosstrack interferograms and do hence not necessarily require coherence between the different passes. Further information can be gained from an evaluation of coherence changes between different passes, potentially augmented by polarimetric information. This could for instance reveal even slight changes in the soil and vegetation structure reflecting vegetation growth and loss, freezing and thawing, fire destruction, human activities, and so on. TanDEM-X enables hence the entry into a new era of interferometric (and tomographic) processing techniques as it was ERS-1/2 for the development of classical repeat-pass SAR interferometry.

\section{Polarimetric SAR Interferometry}

Polarimetric SAR interferometry combines interferometric with polarimetric measurements to gain additional information from semi-transparent volume scatterers [6]. This allows e.g. for the extraction of important biophysical parameters like vegetation density and vegetation height. Fully polarimetric operation uses the split antenna and is susceptible to ambiguities. This can be avoided by reducing the processed azimuth bandwidth and/or by limiting the swath width. Fig. 5 illustrates the achievable performance of a simulated scenario for TanDEM-X. This analysis is based on the Random Volume over Ground (RVoG) model [6] assuming a vegetation layer with a height of $0.6 \mathrm{~m}$ and an extinction coefficient of $10 \mathrm{~dB} / \mathrm{m}$. The dashed line indicates the height variation of the interferometric phase centre with different polarisations (corresponding to a variation of $\mu$ on the abscissa). The green tube shows the height errors due to volume decorrelation for an effective baseline of $5 \mathrm{~km}$ and an independent post-spacing of $30 \mathrm{~m} \times 30 \mathrm{~m}$. The blue tube shows additional errors due to the limited system accuracy and the red tube indicates potential

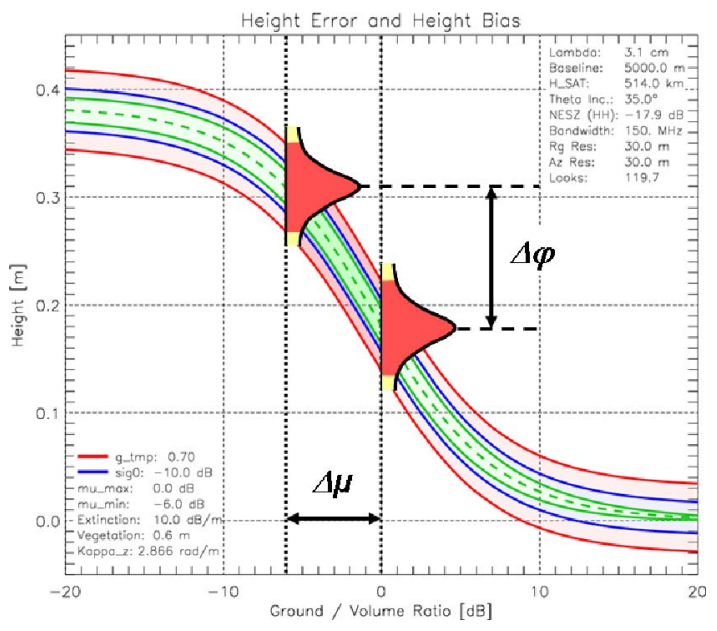

Fig. 5: Vertical separation of interferometric phase centers in TanDEM-X as a function of the ground-to-volume scattering ratio $\mu$ (cf. [7]). The vegetation height is $0.6 \mathrm{~m}$, the ground-to-volume ratio $\mu$ varies from $-6 \mathrm{~dB}$ to $0 \mathrm{~dB}$ and the extinction is $10 \mathrm{~dB} / \mathrm{m}$ 
errors in case of temporal decorrelation caused by a possible along-track separation between the two satellites (here: $\gamma_{\text {Temp }}=$ $0.7)$. The performance analysis predicts a sufficient phase centre separation to enable a successful retrieval of important vegetation parameters like volume height, extinction, etc. TanDEM-X will be the first mission to demonstrate this technique in a single-pass data acquisition mode.

\section{Bistatic SAR Imaging}

Bistatic SAR imaging provides additional observables for the extraction of important scene and target parameters [8]. TanDEM-X allows for the simultaneous acquisition of bistatic and monostatic images in a single data take to obtain a highly informative set of multi-angle observations. A quantitative evaluation of the bistatic radar cross-section (RCS) and a comparison with its monostatic equivalents facilitates the detection and recognition of targets. The segmentation and classification in radar images is expected to be substantially improved by comparing the spatial statistics of mono- and bistatic scattering coefficients. This is also supported by the joint airborne bistatic radar experiments performed by DLR and ONERA, which revealed significant changes of the scattering behaviour for both artificial and natural targets even in case of rather small bistatic angles [9]. A joint evaluation of mono- and bistatic SAR images could furthermore be used to isolate different scattering mechanisms, like e.g. a distinction between highly directive dihedral returns from more isotropic volume scattering. Bistatic SAR imaging has moreover potential for the retrieval of sea state parameters, the estimation of surface roughness and terrain slope, as well as stereogrammetric, meteorological and atmospheric applications. Innovative processing algorithms will be required to exploit all these capabilities. The bistatic data acquired with TanDEM-X will hence provide a unique data source to improve our understanding of bistatic imaging and its exploitation for future remote sensing applications. Data takes with large bistatic angles are planned at the beginning and at the end of the TanDEM-X mission where the satellites are separated from each other by several tenths of kilometers.

\section{E. Digital Beamforming and Super Resolution}

Digital Beamforming combines the RF signals from a set of small non-directional antennas to simulate a large directional antenna. Due to the split antennas and dual receiver channels of TSX and TDX, four phase centers can be obtained in a tandem mode. An appropriate combination of the multiple Rx signals enables then an efficient suppression of azimuth ambiguities [11]. By this, it is possible to demonstrate the capabilities of high resolution wide swath SAR imaging. This technique could also be of interest for advanced interferometric SAR modes like the alternating bistatic mode where it allows for a reduction of the PRF, thereby resolving potential timing and ambiguity conflicts. TanDEM-X will be the first configuration that demonstrates this highly innovative technique from space.

Another promising technique is super resolution. This technique exploits the fact that the signals received by the two satellites have different aspect angles for each scattering point on the ground. In consequence, the two ground range and/or azimuth spectra are shifted relative to each other. A coherent combination of the signals yields then a wider spectrum which corresponds to an improved spatial resolution. This technique requires a cross-track and/or along-track separation in the order of 5 to $10 \mathrm{~km}$. Such baselines will be available in later mission phases after the standard DEM data acquisition has been completed.

\section{CONCLUSIONS}

TanDEM-X is a highly innovative bistatic single-pass SAR interferometer which allows for the acquisition of unique remote sensing data products. The achievable height accuracy for global DEM generation with TanDEM-X is mainly limited by the height of ambiguity that can finally be processed during phase unwrapping. The standard HRTI-3 DEM is a reasonable compromise between performance, processing, and data acquisition effort. A mission scenario has been developed which enables the acquisition of a global HRTI-3 DEM within less than three years [5]. This concept includes several data takes with different baselines, different incident angles, and data takes from ascending and descending orbits to deal with difficult terrain like mountains, valleys, tall vegetation, etc. The TanDEM-X mission concept allocates also sufficient acquisition time and satellite resources to secondary mission goals like along-track interferometry or the demonstration of new bistatic radar techniques.

In 2006, the TanDEM-X mission has been approved for realization by means of a public private partnership between the German Aerospace Center (DLR) and EADS Astrium GmbH. The launch of the TanDEM-X satellite is planned for spring 2009 which ensures at least three years of joint operation with the TerraSAR-X satellite. Current work includes the preparation of a detailed data acquisition plan which has also to take into account potential conflicts between the TerraSAR-X and the TanDEM-X mission, the development and analysis of a calibration concept [12], the design of a multibaseline InSAR processor, the compilation of a science plan as well as performance investigations for the other innovative TanDEM-X imaging modes.

\section{REFERENCES}

[1] A. Moreira, G. Krieger, I. Hajnsek, M. Werner, D. Hounam, S. Riegger, and E. Settelmeyer, "TanDEM-X: A TerraSAR-X Add-On Satellite for SinglePass SAR Interferometry," in Proc. IGARSS, Anchorage, USA, 2004.

[2] M. Stangl, R. Werninghaus, B. Schweizer, C. Fischer, M. Brandfass, J. Mittermayer, and H. Breit, "TerraSAR-X technologies and first results," IEE Proceedings - Radar, Sonar and Navigation, vol. 153, pp. 86-95, 2006

[3] G. Krieger and M. Younis, "Impact of Oscillator Noise in Bistatic and Multistatic SAR," IEEE Geoscience and Remote Sensing Letters, vol. 3, pp. 424- 428,2006

[4] M. Younis, R. Metzig, and G. Krieger, "Performance Prediction of a Phase Synchronization Link for Bistatic SAR," IEEE Geoscience and Remote Sensing Letters, vol. 3, pp. 429-433, 2006.

[5] G. Krieger, A. Moreira, H. Fiedler, I. Hajnsek, M. Werner, M. Younis, M. Zink, "TanDEM-X: A Satellite Formation for High Resolution SAR Interferometry," to appear in IEEE Transactions on Geoscience and Remote Sensing, 2007.

[6] S.R. Cloude and K.P. Papathanassiou, "Polarimetric SAR interferometry," IEEE Transactions on Geoscience and Remote Sensing, vol. 36, pp. 1551$1565,1998$.

[7] G. Krieger, K. Papathanassiou, S. Cloude, "Spaceborne Polarimetric SAR Interferometry: Performance Analysis and Mission Concepts," EURASIP Journal on Applied Signal Processing, vol. 20, pp. 3272-3292, 2005.

[8] G. Krieger and A. Moreira, "Spaceborne Bi- and Multistatic SAR: Potential and Challenges," IEE Proceedings - Radar, Sonar and Navigation, vol. 153, pp. 184-198, 2006.

[9] P. Dubois-Fernandez, H. Cantalloube, B. Vaizan, G. Krieger, R. Horn, M. Wendler, and V. Giroux, "ONERA-DLR bistatic SAR campaign: Planning, data acquisition, and first analysis of bistatic scattering behavior of natural and urban targets," IEE Proceedings - Radar, Sonar and Navigation, vol. 153, pp. 214-223, 2006.

[10] J. Mittermayer, H. Runge, "Conceptual Studies for Exploiting the TerraSARX Dual Receive Antenna," Proc. IGARSS, Toulouse, France, 2003.

[11] G. Krieger, N. Gebert, and A. Moreira, "Unambiguous SAR signal reconstruction from nonuniform displaced phase center sampling," IEEE Geoscience and Remote Sensing Letters, vol. 1, pp. 260-264, 2004.

[12] J. Hueso González, M. Bachmann, H. Fiedler, S. Huber, G. Krieger, M. Zink, B. Wessel, "Development of TanDEM-X DEM Calibration Concept," in Proc. EURAD, Munich, Germany, 2007. 